\title{
Strong focusing gradient in a linear active plasma lens
}

\author{
K. N. Sjobak $\odot,{ }^{1,2,{ }^{*}}$ E. Adliø, ${ }^{1}$ R. Corsini, ${ }^{2}$ W. Farabolini, ${ }^{2}$ G. Boyle $\odot,{ }^{3}$ C. A. Lindstrøm $\odot,{ }^{3}$ \\ M. Meisel, ${ }^{3}$ J. Osterhoff $\odot,{ }^{3}$ J.-H. Röckemann $\odot,{ }^{3}$ L. Schaper, ${ }^{3}$ and A. E. Dyson ${ }^{4}$ \\ ${ }^{1}$ Department of Physics, University of Oslo, 0316 Oslo, Norway \\ ${ }^{2}$ CERN, CH-1211 Geneva 23, Switzerland \\ ${ }^{3}$ Deutsches Elektronen-Synchrotron DESY, Notkestraße 85, 22607 Hamburg, Germany \\ ${ }^{4}$ Department of Physics, Clarendon Laboratory, University of Oxford, \\ Parks Road, Oxford OXI 3PU, United Kingdom
}

(Received 30 September 2021; accepted 10 December 2021; published 29 December 2021)

\begin{abstract}
Active plasma lenses are compact devices developed as a promising beam-focusing alternative for charged particle beams, capable of short focal lengths for high-energy beams. We have previously shown that linear magnetic fields with gradients of around $0.3 \mathrm{kT} / \mathrm{m}$ can be achieved in argon-filled plasma lenses that preserve beam emittance [C.A. Lindstrøm et al., Phys. Rev. Lett. 121, 194801 (2018)]. Here we show that with argon in a $500 \mu \mathrm{m}$ diameter capillary, the fields are still linear with a focusing gradient of $3.6 \mathrm{kT} / \mathrm{m}$, which is an order of magnitude higher than the gradients of quadrupole magnets. The current pulses that generate the magnetic field are provided by compact Marx banks, and are highly repeatable. The demonstrated operation with simultaneously high-gradient, linear fields and good repeatability establish active plasma lenses as an ideal device for pulsed particle beam applications requiring very high focusing gradients that are uniform throughout the lens aperture.
\end{abstract}

DOI: 10.1103/PhysRevAccelBeams.24.121306

Plasma-based technology promises to pave the way for more compact particle accelerators [1]. While much research has been directed toward the use of plasma technology for compact acceleration [2-4], there has been less effort directed at compactifying the focusing elements of the accelerator, which is also crucial for achieving compact machines. The development of plasma lenses, as discussed here, may establish a superior beam-focusing alternative for charged particle beams in high energy physics applications, as well as for other demanding pulsed-beam applications such as medical applications or photon sources [5-7].

Quadrupole magnetic lenses are the conventional choice for focusing of high-energy charged beams [[8] Section 7.2]. Permanent quadrupole magnets can be made compact and precise [9], but the focusing gradient is currently limited to a few $100 \mathrm{~T} / \mathrm{m}[9,10]$, and they have a limited tuning range. Furthermore, a quadrupole defocuses the beam in the plane orthogonal to the focusing plane, requiring a lattice of several magnets to achieve an overall focusing effect.

Active plasma lenses [11,12] (APLs) generate a focusing magnetic field by passing a strong current through a plasma

*k.n.sjobak@fys.uio.no

Published by the American Physical Society under the terms of the Creative Commons Attribution 4.0 International license. Further distribution of this work must maintain attribution to the author(s) and the published article's title, journal citation, and DOI. that is parallel to and overlapping with the beam axis, creating an axisymmetric focusing force. Axisymmetric focusing is particularly important for capturing and refocusing beams with high divergence and energy spread, e.g., a beam produced in a plasma-wakefield accelerator $[4,13,14]$. Implementations of APLs using discharge capillary technology [15] have recently been developed $[11,16]$, and first uses as optical elements for accelerator research applications are reported [17-19].

A challenge for APLs are different sources of nonlinearity in the fields, mainly due to nonuniform plasma heating [20] and wakefields [21]. Recent work has shown that these nonlinearities can be avoided, and that active plasma lensing with linear focusing forces can be achieved $[17,22]$, which is necessary to preserve beam emittance. Historically, very high gradients have been reached in z-pinch-discharge APLs [23,24], however these devices did not produce a linear focusing magnetic field. More recently gradients of $\approx 3.5 \mathrm{kT} / \mathrm{m}$ have been demonstrated without pinching, however this was in a nonlinear hydrogen lens with a small diameter of $250 \mu \mathrm{m}$ [11].

When increasing the magnetic field gradient in an APL, several technical and physical challenges must be overcome in order to produce a useful device. Our experiment improves the demonstrated gradients by developing compact high voltage sources with fast and reproducible pulses, while still avoiding both the thermal nonlinearities seen for light gases such as helium [20,25-27], and the pinching of the field-producing current stream in the plasma [28]. 
The results demonstrate that APLs may repeatedly operate at multi-kT/m gradients, outperforming quadrupole magnets, and at the same time provide linear focusing forces.

Operating at high focusing gradients reduces the size of the focusing device, which can be important for maintaining the accelerating gradient in plasma-wakefield accelerators [29]. Reducing the length of the plasma is also expected to reduce the emittance growth in an APL from plasma wakefields, especially near the lens exit because the beam converges less within the lens [30].

Our results therefore represent a key step for establishing the APL as a versatile and useful device for compact, pulsed-beam applications.

\section{EXPERIMENTAL SETUP}

The CLEAR Plasma Lens Experiment [16,22,31] is installed at the CLEAR user facility at CERN [32-34], using a $200 \mathrm{MeV}$ linear electron accelerator to produce beams with a wide range of possible parameters.

The principle of the experiment is illustrated in Fig. 1. First, a strongly focused beam enters the capillary, in order to probe the field in a small region. This beam is then deflected towards the center of the lens by the focusing force. After exiting the lens, there is a short drift before the beam impinges on an optical transition radiation (OTR) screen mounted at a $45^{\circ}$ angle to the beam, used to measure the beam position. This screen is shielded from the light emitted from the plasma with a thin metallized polymer foil; this limits us to only measuring vertical beam deflection, as otherwise the distance between the foil and the screen changes as the beam moves across it, causing a change in the apparent beam shape [35].

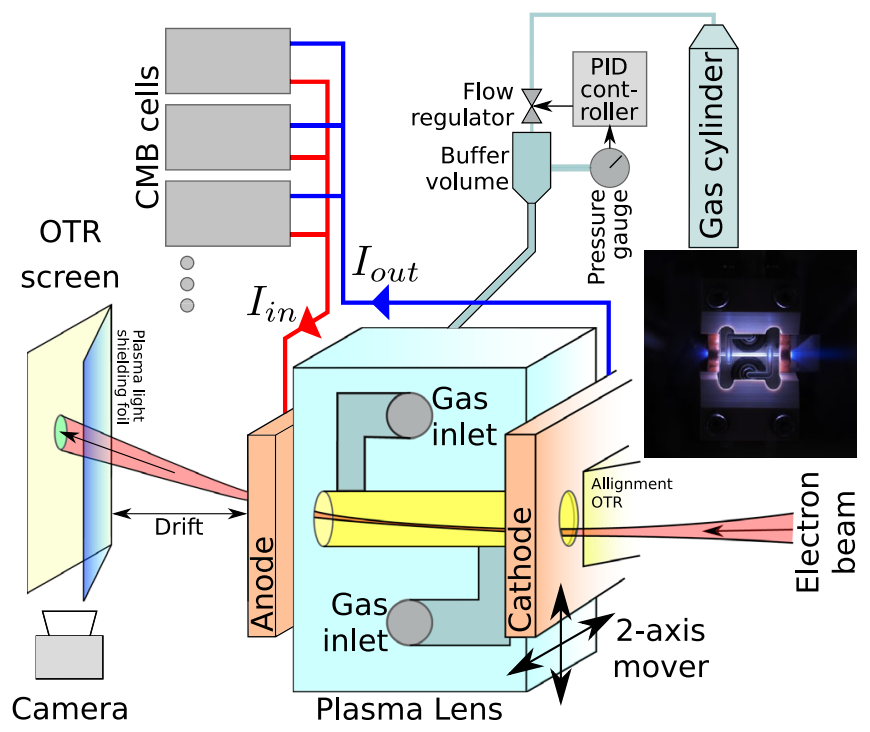

FIG. 1. Overview of the most relevant components of the CLEAR Plasma Lens Experiment and the path of the beam. Insert: Plasma lens capillary during a discharge.
A small OTR screen is mounted on the upstream (cathode) electrode of the plasma lens, in order to aid focusing the beam and aligning the lens to the beam, as shown in Fig. 1. The electron beam was focused down to a horizontal $\times$ vertical size of $44 \times 52 \mu \mathrm{m}$ root mean square (rms) as measured on this alignment OTR, and by the falloff of beam intensity as a function of lens position when scanning the aperture edge over the beam. This is small enough for the beam to pass cleanly through the capillary containing the current-carrying plasma and to sample the field locally.

In order to measure the magnetic field in the lens as a function of position, the lens is moved transversely relative to the beam. The beam position on the OTR screen behind the lens is then measured as a function of the lens position, and compared to the beam position without discharge in order to measure the deflection. The magnetic field gradient can then be reconstructed as discussed in Sec. II C. Note that due to the very high gradients, the plasma lens must be treated as a thick lens because the focal length is on the order of the length of the lens, such that an initially parallel beam would converge $\approx 20 \mathrm{~mm}$ after the lens entrance.

Furthermore, the gradient enhancement was measured as a function of time, by holding the lens at a known position and changing the relative timing of the lens with respect to the beam. The gradient enhancement was then found by comparing the deflection expected from the current measurement with the one observed on the screen, as described in Sec. II D.

\section{A. Discharge capillary}

The discharges are contained within a sapphire capillary, as shown in the insert in Fig. 1. For the experiments reported in this paper, a $15 \mathrm{~mm}$ long capillary with a nominal diameter of $500 \mu \mathrm{m}$ was used. It is made by milling out a semicircular groove in two sapphire plates which are then pressed together.

For small-diameter capillaries, the actual manufactured capillary may not be perfectly round. This breaks the symmetry between the current distribution in the two planes, causing an astigmatism in the focusing effect of the lens. This can be compensated for when finding the gradient and gradient enhancement from the deflection and current measurements as discussed in Sec. II. For the capillary used in this paper, the diameters were measured using optical profilometry to be $525 \mu \mathrm{m}$ horizontally and $543 \mu \mathrm{m}$ vertically. This causes a difference in the measured quantities, however this is within the statistical errors of the measurements. The correction was therefore omitted in order to reduce the complexity of the measurement, simply using the mean radius of $R=267 \mu \mathrm{m}$.

The capillary is supplied with gas at an adjustable pressure through two inlet channels, giving a flat initial pressure profile between these channels. In order to maintain the accelerator vacuum, a turbo-pump removes 
the gas escaping from the capillary openings, and a $3 \mu \mathrm{m}$ Mylar beam window is used to separate the plasma lens chamber from the upstream accelerator vacuum.

The breakdown voltage and drive current pulse is supplied via a set of copper electrodes on each end of the capillary, with holes that allow the beam to pass in and out of the channel.

\section{B. Compact Marx bank}

The high currents needed in order to achieve the high focussing gradients were made possible by connecting several compact Marx bank (CMB) cells [36] in parallel, reported here for the first time. Up to $8 \mathrm{CMB}$ cells were used and details of cell triggering and pulse jitter are considered below. Each CMB cell consists of a 10-stage ladder network with $2 \times 22 \mathrm{nF}$ capacitor rungs initially charged to $2.0 \mathrm{kV}$. This gives a floating initial discharge voltage of $20 \mathrm{kV}$ with an effective cell capacitance of $4.4 \mathrm{nF}$, corresponding to about $1 \mathrm{~J}$ of pulse energy per cell. Using a floating voltage significantly inhibits breakdown to the vacuum chamber walls. This voltage is higher than that strictly needed for gas breakdown, to ensure a rapid onset of the discharge within a few ns of the voltage pulse being applied. Once the plasma is formed the current pulse starts to rise, mainly constrained by circuit inductance.

Each cell is separately triggerable from its own trigger unit, giving a $-1.5 \mathrm{kV}$ trigger pulse that is transformed by an onboard minitransformer to around $-5 \mathrm{kV}$ with a sub 20 ns risetime. This causes the first stage spark gap (SG1) to fire, resulting in an avalanche voltage pulse that cascades down the CMB. Typically, SG1 fires at around half peak trigger voltage, and the fast risetime means that the SG1 gap separation need only be roughly set for all cells to fire within about a $10 \mathrm{~ns}$ intrinsic delay relative to each other.

The $2 \mathrm{kV}$ charging voltage to each cell is remotely switchable with unwanted cells left uncharged, allowing for some degree of coarse current adjustment. The charging resistors are chosen so that each cell reaches its maximum charging voltage every $1.2 \mathrm{~s}$, which is the beam rate of the CLEAR machine. The final HV $10 \Omega$ resistor protects the cell in case of an external short circuit.

The CMB cells are arranged on a single chassis in banks of four and enclosed in a $210 \times 297 \times 50 \mathrm{~mm}$ box (same footprint as an A4 sheet of paper), placed a within a few $\mathrm{cm}$ from the plasma lens. The cells provide high-current pulses with sub- $\mu$ s duration and fast rise time as shown in Fig. 2, appropriate for driving a plasma lens while limiting the total amount of energy deposited in each pulse to reduce capillary damage by heating. The close proximity of the mounting eliminates the need for impedance matching.

Combining several cells in parallel brings a number of advantages. Firstly the total current is increased according to the number of cells used and thus the gradient. Simultaneously the jitter in both timing and peak current is reduced. Furthermore, the reliability is also increased,

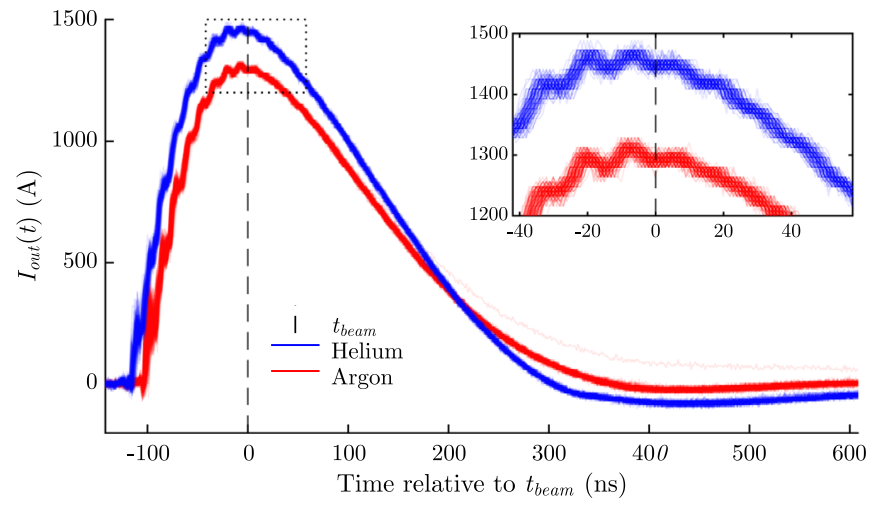

FIG. 2. Compact Marx bank current traces for the offset scans in helium and argon that are shown in Figs. 6 (180 traces) and 7 (136 traces).

as the current drawn from each cell is reduced to below $200 \mathrm{~A}$, and in the case of the loss of a single cell the lens can still operate in a degraded peak current mode.

The current output of the CMBs are measured by two current-pulse transformers; one on each cable in order to detect large losses to ground. The current traces during the offset scans described in the next section are shown in Fig. 2. This shows that the current rises from 0 to peak in $\approx 100 \mathrm{~ns}$, has a flat-top of a few 10 s of ns, before it falls off in $\approx 300$ ns. For both argon and helium, the incoming and outgoing currents at the time of beam arrival are identical, and their standard deviation are both equal to the sampling resolution which is $8 \mathrm{~A}$. In summary, the current pulses are generally highly reproducible.

\section{RECONSTRUCTION OF MAGNETIC-FIELD GRADIENT}

In order to determine the magnetic field gradient and profile from the beam deflection measurements, models of the effect on the beam from the field are needed. This needs to take into account the thick lens effect which is caused by transverse deflection of the beam inside the lens due the strong magnetic field, and current density nonuniformities caused by temperature gradients in the plasma.

\section{A. Magnetic field in capillaries}

The magnetic field in a cylindrical active plasma lens can be found through the application of Ampère's law on loops of constant radius $r=\sqrt{x^{2}+y^{2}}$ :

$$
B_{\theta}(r)=\frac{\mu_{0}}{2 \pi r} \int_{0}^{r} J\left(r^{\prime}\right) r^{\prime} \mathrm{d} r^{\prime}
$$

For the case of a uniform current density, the expected field gradient is then

$$
g=\frac{\mathrm{d} B_{\theta}}{\mathrm{d} r}=\frac{\mu_{0} I}{2 \pi R^{2}}=\frac{B_{\text {surf }}}{R} .
$$




\section{B. $J \propto T^{3 / 2}$ model for thermal nonuniformity}

The JT-model [20,22,25] describes how a gradient nonuniformity can be caused by a radial variation of the electron temperature $T_{e}=A u(\xi)^{2 / 7}$ in the capillary, where $A$ is a constant given by the capillary size and plasma parameters, $\xi=r / R$, and $u(\xi)$ is a solution to the heat flow equation

$$
\frac{1}{\xi} \frac{\mathrm{d}}{\mathrm{d} \xi}\left(\xi \frac{\mathrm{d} u}{\mathrm{~d} \xi}\right)=-u^{3 / 7}
$$

This causes a variation of the conductivity $\sigma=\sigma_{0} T_{e}^{3 / 2}$, which causes the nonuniformity of the current density $J=\sigma E$, creating the nonlinearity of $B_{\theta}(\xi)$. Combining these gives $J=\sigma_{0} E A^{3 / 2} u^{3 / 7}$, where the unknown constant $\sigma_{0} E A^{3 / 2}$ can be determined through conservation of current $I=\int_{0}^{1} J(\xi) \mathrm{d} \xi$, and $u(\xi)$ through solving Eq. (3). The current density is then given as

$$
J(\xi)=\frac{I u(\xi)^{3 / 7}}{2 \pi a b \int_{0}^{1} u^{3 / 7} \xi \mathrm{d} \xi},
$$

and the magnetic field can be determined through Eq. (1).

\section{Magnetic-field-gradient measurement technique considering thick-lens effect}

The measurement of the magnetic gradient is based on measuring the deflection of a particle beam passing through the lens, as illustrated in Fig. 3. The position of the beam on the downstream profile monitor is compared between the case where the plasma lens is on and off, giving a deflection $\Delta y=y_{f}-y_{i}$.

Because the focal length is short compared to the length of the lens, the thick-lens effect must be correctly taken into

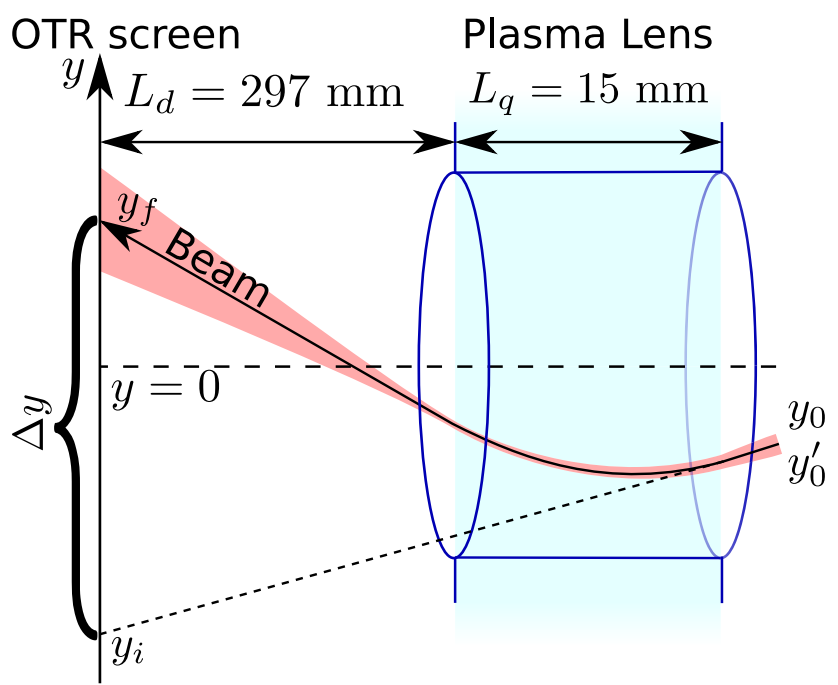

FIG. 3. Definition of variables used in discussion of thick-lens gradient reconstruction. account to find the gradient. For a lens with uniform current density, the position on the screen is given by

$$
\begin{aligned}
y_{f}= & y_{0}\left(\cos \left(\sqrt{K} L_{q}\right)-\sqrt{K} L_{d} \sin \left(\sqrt{K} L_{q}\right)\right) \\
& +y_{0}^{\prime}\left(\sin \left(\sqrt{K} L_{q}\right) / \sqrt{K}+L_{d} \cos \left(\sqrt{K} L_{q}\right)\right) \\
\equiv & y_{0} A(K)+y_{0}^{\prime} B(K),
\end{aligned}
$$

where the focusing strength is

$$
K=\frac{\mathrm{d} B_{y}}{\mathrm{~d} x} \frac{e}{p_{0}}
$$

where $p_{0}$ is the reference momentum of the beam and $e$ is the particle (electron) charge. The deflection on the screen is thus given as

$$
\begin{aligned}
\Delta y & =y_{0} A(K)+y_{c}-y_{i}\left(y_{0}\right) \\
& =y_{f}\left(K, y_{0}, y_{0}^{\prime}\right)-y_{i}\left(y_{0}, y_{0}^{\prime}\right),
\end{aligned}
$$

where $y_{c} \equiv y_{0}^{\prime} B(K)$ is a constant term due to the initial beam angle $y_{0}^{\prime}$, and $y_{i}=y_{0}+y_{0}^{\prime}\left(L_{q}+L_{d}\right)$ is the position on the screen when the lens is switched off and $K \rightarrow 0$.

In order to find the strength $K$ and thus the gradient seen by the beam $g_{\text {beam }}=\frac{\mathrm{d} B_{x}}{\mathrm{~d} y}$, we measure $\mathrm{d} \Delta y / \mathrm{d} y_{0}$, the derivative deflection as a function of the initial position, with a linear fit to offsets. From this, the gradient is determined by $K$ by numerically finding the root of

$$
\frac{\mathrm{d} \Delta y}{\mathrm{~d} y_{0}}-A(K)+1=0
$$

The method for gradient measurement in a thick lens was experimentally verified by reversing the current direction in the lens, running it in the defocusing mode with argon gas. In this case, the beam is pushed outwards while traversing the lens, increasing the kick instead of decreasing it. The same focusing gradient was then found in the reversed case as in the normal case, where the other parameters were kept constant.

In order to apply this method to a nonlinear thick elliptical lens with a field distribution given by the JT-model [Eqs. (4) and (1)], a numerical tracking method is used instead of Eq. (5) to track the beam centroid through the lens. For this, the lens is sliced into $1 \mathrm{~mm}$ thick slices, and the field seen by the beam centroid is computed at each slice. The computed deflection as a function of the lens position is then fitted to the data, using the initial angle $y_{0}^{\prime}$ and the central normalized temperature $u(0)$ as free parameters, and the measured current and capillary radius as input. Due to thick-lens effects, it is expected that $\Delta y$ at the aperture boundary depends on the current distribution inside the lens, even if the magnetic field $B_{\theta}(\xi=1)$ is independent of the current distribution. 
Note that in the experiment the lens itself was moved relative to the beam, which is mathematically equivalent to shifting both $y_{0}$ and $y_{f}$ in the opposite direction of the lens movement. This changes Eq. (5) to

$$
y_{f}=y_{0}\left(A(K)-y_{L}\right)+y_{0}^{\prime} B(K)+y_{L},
$$

where $y_{L}$ is the lens offset. Furthermore, the equivalent of Eq. (8) uses a derivative with respect to $y_{L}$ instead of $y_{0}$.

\section{Field-enhancement time development considering thick-lens effect}

For measuring how the gradient enhancement

$$
g_{\text {enh }}=\frac{g_{\text {beam }}}{g_{\text {uniform }}}
$$

develops in time, timing scans are used. In these, the trigger of the CMBs are scanned relative to the time of arrival of the beam, so that the beam probes the discharge at different times. For these experiments, the position of the lens and the initial beam position is kept constant; if assuming a linear lens the centroid position on the final screen is then given by Eq. (5). This depends on three parameters: The focusing strength $K$, and the initial beam position $y_{0}$ and angle $y_{0}^{\prime}$ relative to the lens axis. In the experiment $y_{0}$ is known from the lens mover position, relatively to the center which is found as the lens position where the centroid kick is zero, and verified as being in the center of the lens aperture. This procedure works well as long as $y_{0}^{\prime}$ is small, which is ensured during beam setup by varying the beam angle in order to maximize the apparent beam aperture through the capillary. However, it is not possible to completely eliminate this angle, which is therefore estimated from the data and taken into account.

By measuring the beam deflection $\Delta y$ at several $K(I(t))$ and assuming that $g_{\mathrm{enh}}$ is constant and $y_{0}=-150 \mu \mathrm{m}$, the initial beam angle and the overall gradient enhancement can be found. This is achieved by a nonlinear least-squares fit to Eq. (5) as a function of $I$, shown in Fig. 4, giving $y_{0}^{\prime}=0.85 \mathrm{mrad}$ and $g_{\mathrm{enh}}=0.96$. The angle is also small enough that the reduction of apparent aperture over the length of the $15 \mathrm{~mm}$ long capillary would be barely noticeable. The method for finding the beam angle is also robust as if locking $g_{\text {enh }}=1$ and instead fitting $y_{0}$ and $y_{0}^{\prime}$, a position of $-145 \mu \mathrm{m}$ and an angle of $0.83 \mathrm{mrad}$ is found.

Once the initial position and angle is established, the actual strength $K$ and thus $g_{\text {beam }}$ can be found for each timepoint by solving Eq. (5) for $K$ and computing $g_{\text {beam }}$, as discussed in Sec. III. Comparing this to the expected gradient in case of a uniform current then gives an estimate of the gradient enhancement $g_{\mathrm{enh}}$ as a function of time, as shown in Fig. 5.

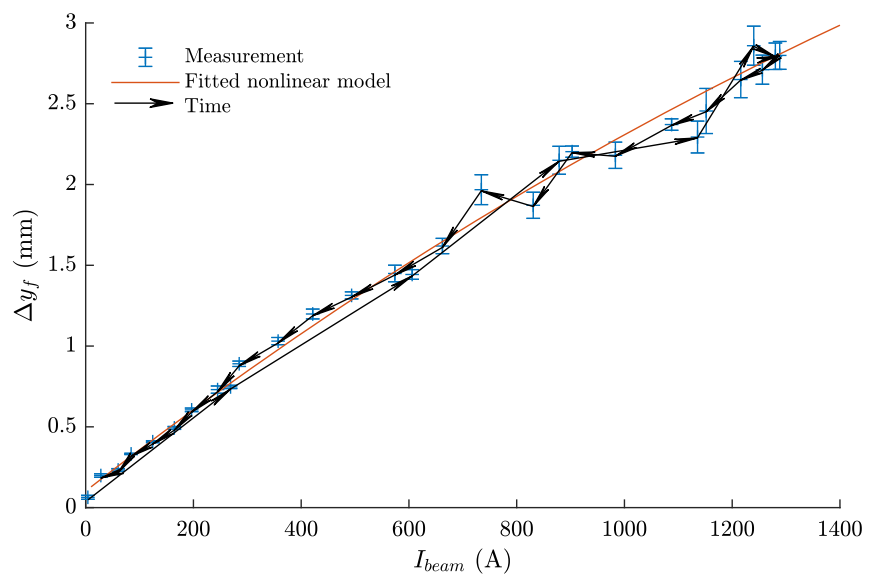

FIG. 4. Beam deflection as a function of current, and a nonlinear fit with used to estimate $g_{\mathrm{enh}}$ and $y_{0}^{\prime}$, assuming $y_{0}=-150 \mu \mathrm{m}$,. The "time" arrows points toward measurements where the beam arrives later relative to the start of the discharge, i.e., from left to right in Figs. 2 and 5.

\section{MAGNETIC-FIELD-GRADIENT MEASUREMENTS}

The magnetic-field-gradient measurements were done in both argon and helium, and the lens was operated at a neutral gas pressure in the buffer volume of 25 mbar and 55 mbar respectively. These were the minimum values for stable operation of the discharge in the $0.5 \mathrm{~mm}$ capillary used here. The expected gas pressure $P$ in the capillary is then approximately 8 mbar for argon and 17 mbar for helium [22]. Performing the gradient measurements in both gas species was done in order to be able to compare the gradient enhancement between the gases and with earlier measurements. Using two gases also serves as a check on the gradient enhancement measurement, since a notable enhancement is expected for a lighter gas.

The beam energy was $195 \mathrm{MeV}$, using three bunches for a total train length of $2 \mathrm{~ns}$, each with a bunch length 4 ps rms, and a total charge of $200 \mathrm{pC}$. The beam size on the screen soon after the current had returned to zero was the same as before plasma ignition, indicating negligible influence from plasma wakefields. The transverse gradient from plasma wakefields, which are centered on the beam, were estimated to have a strength equivalent to approximately $30 \mathrm{~T} / \mathrm{m}$ [21].

This relatively high beam charge was necessary to be able to measure the beam position on the OTR screen in spite of the strong overfocusing in the lens, causing a diffuse beam with a size of $\approx 1.4 \mathrm{~mm}$ at this location. The large size made the accurate reconstruction of the beam position more difficult, contributing to the error bars seen in Figs. 6 and 7, which indicate the standard error of the mean reconstructed beam position. The field was measured only in the central $400 \mu \mathrm{m}$ of the capillary; in the last $\approx 50 \mu \mathrm{m}$ from the edge the beam was deflected outside the screen. 


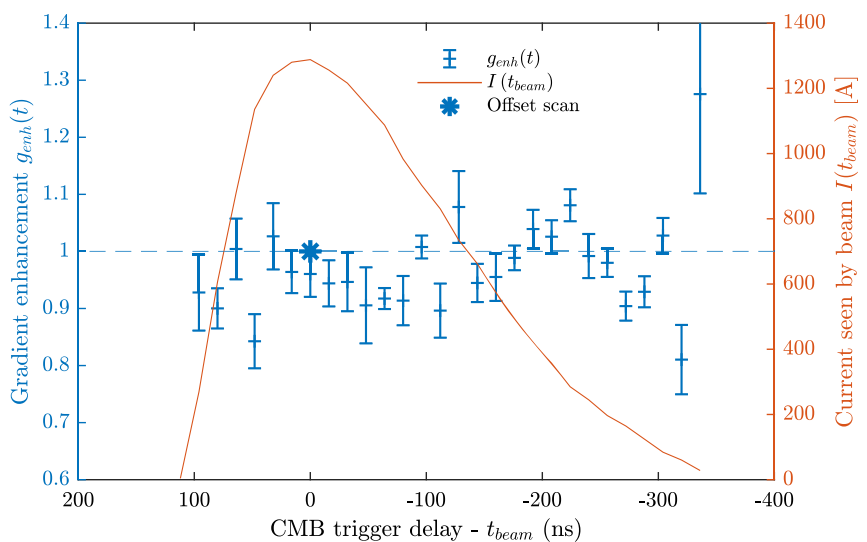

FIG. 5. Estimated gradient enhancement as a function of time at a fixed offset $\left(y_{0}=-150 \mu \mathrm{m}\right)$ in argon. The result from the offset scan shown in Fig. 6 is also indicated.

The beam displacement as function of position for argon is shown in Fig. 6, and fitted as described in Sec. II C. The measured gradient from a linear fit was found to be $3.64 \pm 0.07 \mathrm{kT} / \mathrm{m}$, where the error estimate is the standard deviation due to the uncertainty of the position measurements. No evidence of a gradient enhancement [20] near the center was observed. The gradient near the center was evaluated by fitting a nonlinear JT-model to the whole scan, with the total current as an input parameter.

The measurement of gradient enhancement as a function of time for argon is shown in Fig. 5, using the beam angle and method described in Sec. II D. This shows that the gradient enhancement remained constant throughout the discharge; the weighted average being $0.97 \pm 0.08$. The error bars on the gradient enhancement is the estimated standard deviation derived from a combination of $1 \mathrm{~ns}$ uncertainty on the time of sampling the current for the calculation of $g_{\text {uniform }}$, and the uncertainty on the mean beam position on the screen at each timing for the calculation of $g_{\text {beam }}$.

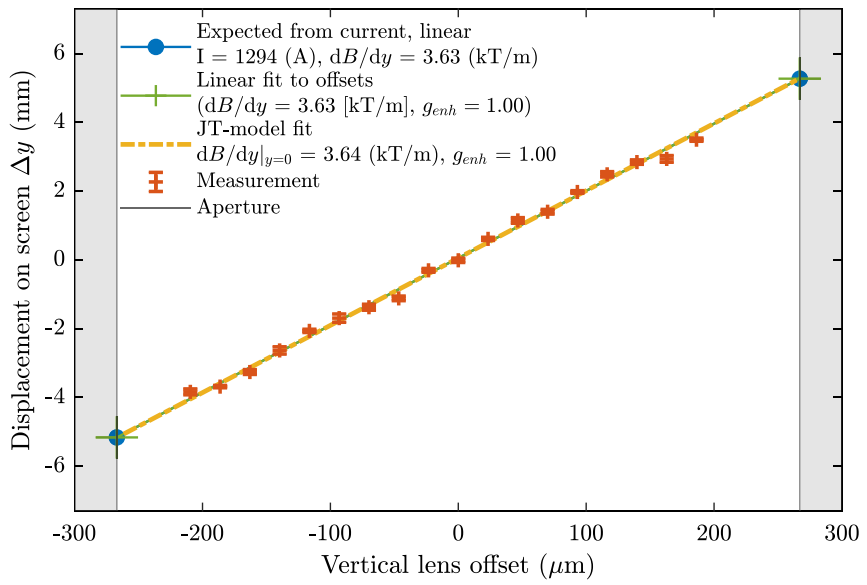

FIG. 6. Beam displacement as a function of lens offset in argon on peak current, for measuring the field gradient.

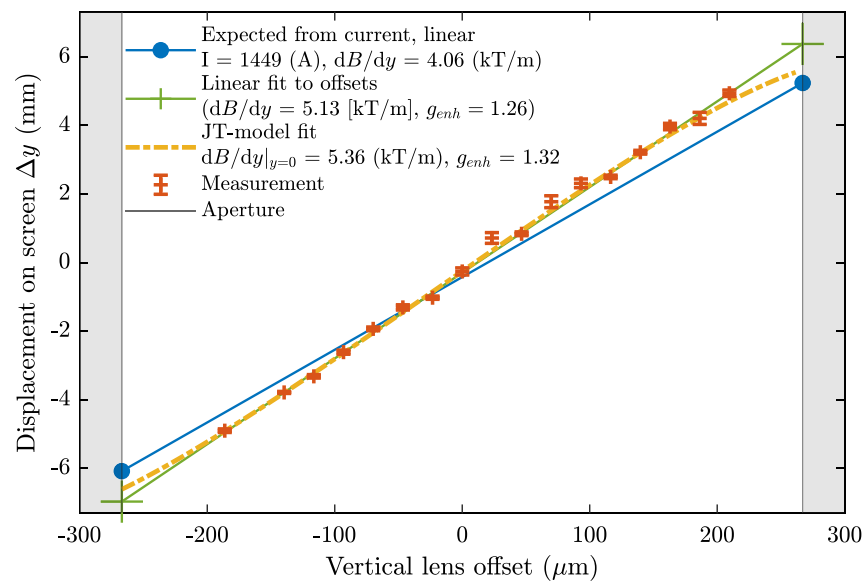

FIG. 7. Beam displacement as a function of lens offset in helium on peak current, for measuring the field gradient.

For helium the beam displacement as function of position is shown in Fig. 7, where the observed central gradient was $5.36 \pm 0.13 \mathrm{kT} / \mathrm{m}$. Here the nonlinear model fit found a gradient enhancement near the center of $32 \pm 3 \%$, where the error is the standard deviation due to the uncertainty of the beam position measurements.

\section{DISCUSSION}

The central focusing gradients were obtained directly from the beam deflection measurement, using fits based on the measured current, capillary radius, and mover positions. For argon the results indicate that even at these very high gradients, APLs remain linear throughout the time- and aperture range that we tested, which is a necessary condition for emittance preservation. For helium the observed gradient enhancement factor is consistent with the $34 \%$ found earlier [22] at a lower central gradient of $440 \mathrm{~T} / \mathrm{m}$, using a $1000 \mu \mathrm{m}$ diameter capillary and single $\mathrm{CMB}$ cell producing a peak current of $410 \mathrm{~A}$.

The gradient enhancement factor in argon was also independently measured as a function of time, confirming that it remains constant and close to unity throughout the duration of the discharge. This implies that the gradient can be fine-tuned simply by changing the timing of the discharge, without incurring nonlinearity.

In earlier work [21] it has been shown that beams with high charge density can create waves in the plasma, which in turn may lead to nonlinear fields. Such fields are a source of increased beam emittance. The relative effect of the wakes may be reduced by increasing the plasma lens gradient [30,37], since an increased gradient allows a shorter lens. A shorter lens limits the increase of wakefield while passing through the lens, both directly by a reduction of distance travelled in the plasma, and indirectly because the shortened drift means the beam size is more consistent throughout the lens. Furthermore, a reduced length of the lens will reduce the amount of collisions between the beam 
and the gas, which could be especially important for heavy gasses. Hence our results increase the parameter range where APLs with linear fields can be used.

Another potential source of nonlinearities is the $z$-pinching of the current in the plasma lens, due to the magnetic pressure overcoming the thermal pressure in the plasma, i.e., when

$$
\frac{B^{2}}{2 \mu_{0}}=\frac{\mu_{0} I^{2}}{8 \pi^{2} R^{2}}>n_{0} k T_{e}
$$

where $T_{e}$ is the electron temperature, and $n_{0}$ the electron density. This will tend to concentrate the current near the axis, forming a nonlinear and potentially unstable field [24,28,38]. The well-known "snow plow"-models [23,38-40] assume that the current is concentrated in a thin layer on the outside of the plasma, with a thickness on the order of the skin layer thickness. However in our case, the thickness of the skin layer is on the order of the lens diameter, as also confirmed from the transverse field profile measurements in Sec. III.

This precludes the usability of snowplow-models for the prediction of pinch dynamics in our device, however Eq. (11) still holds, indicating that if the magnetic pressure is large enough, a pinch will eventually occur. For the argon lens at peak current, the static pressure balance of Eq. (11) is maintained if $k T_{e}>14 \mathrm{eV} / z$. Here $z$ is the average ionization level of the plasma, since the electron density $n_{0}$ is proportional to $z P$. As seen in Fig. 6, the field on peak is consistent with a uniform current density through the lens up to $200 \mu \mathrm{m}$, which indicates that no pinch has happened at that point. Potential further studies include field mapping for larger lens diameters to precisely measure the onset of the pinch, as these have larger fields at the aperture if the gradient is similar. This could be a limitation in the useful parameter range for linear active plasma lenses, however it was not reached here.

The group of experimental CMB cells reached very high currents without any major failures. The addition of several of these in parallel tended to stabilize the discharge compared to previous experiments, and reduced the failure rate of the system as less current was drawn from each CMB cell. This principle allows for coarse tunability of the total current and redundancy in the system by overprovisioning the number of cells needed for the nominally required current.

\section{CONCLUSION}

In conclusion, we demonstrate the operation of a compact, linear APL in argon with a focusing gradient of $3.6 \mathrm{kT} / \mathrm{m}$. The absence of a gradient enhancement factor was confirmed with two different measurements techniques. These techniques were especially developed to work with strong lenses that cannot be considered to be thin.

With helium as the fill gas the lens is nonlinear with a central gradient of $5.4 \mathrm{kT} / \mathrm{m}$ and a gradient enhancement factor of $32 \%$. These gradient enhancement factors are consistent with what was previously measured at lower gradients [22]. The lens has a diameter of $500 \mu \mathrm{m}$ and is powered by up to 8 compact Marx banks (CMB) cells connected in parallel.

The use of CMB cells enable us to reach the high currents required to drive the lens, with short-duration pulses. This presents compact high-voltage and highcurrent source that can reach even higher currents by simply adding more cells.

These findings open up the possibility for using APLs in applications that require linear focusing with gradients well beyond those of quadrupole magnets, and in applications that otherwise benefit from a strong axisymmetric linear focusing element.

\section{ACKNOWLEDGMENTS}

We thank CERN for providing beam time at the CLEAR User Facility, and especially A. Gillardi, D. Gamba, A. Curcio, A. Chauchet, S. Curt, G. McMonagle, S. Burger, M. Bergamaschi, T. Lefevre, E. Granados, and H. Panuganti, for making it possible to run the accelerator, and for often lending us a hand and suggestions for improving the experiment. We also thank S. Hooker for suggestions for improving the manuscript. This work was supported by the Research Council of Norway (NFR Grant No. 310713) and the Helmholtz Association of German Research Centers (Grants No. VH-VI-503 and No. ZT-0009).

[1] C. Joshi and T. Katsouleas, Plasma accelerators at the energy frontier and on tabletops, Phys. Today 56, No. 647 (2003).

[2] B. Cros and P. Muggli, Towards a Proposal for an Advanced Linear Collider, Report on the Advanced and Novel Accelerators for High Energy Physics Roadmap Workshop (CERN, Geneva, 2017), ISBN 978-92-9083468-7 (paperback), ISBN 978-92-9083-469-4 (PDF).

[3] D. Schulte, Application of advanced accelerator concepts for colliders, Rev. Accel. Sci. Techol. 09, 209 (2016).

[4] C. A. Lindstrøm, E. Adli, J. M. Allen, J. P. Delahaye, M. J. Hogan, C. Joshi, P. Muggli, T. O. Raubenheimer, and V. Yakimenko, Staging optics considerations for a plasma wakefield acceleration linear collider, Nucl. Instrum. Methods Phys. Res., Sect. A 829, 224 (2016).

[5] C. Joshi, S. Corde, and W. B. Mori, Perspectives on the generation of electron beams from plasma-based accelerators and their near and long term applications, Phys. Plasmas 27, 070602 (2020).

[6] M.E. Couprie, Towards compact Free Electron Laser based on laser plasma accelerators, Nucl. Instrum. Methods Phys. Res., Sect. A 909, 5 (2018).

[7] A. R. Maier, M. Kirchen, and F. Grüner, Brilliant light sources driven by laser-plasma accelerators, in Synchrotron Light Sources and Free-Electron Lasers: Accelerator Physics, Instrumentation and Science Applications, edited by E. J. Jaeschke, S. Khan, J. R. Schneider, and J. B. 
Hastings (Springer International Publishing, Cham, 2020), pp. 245-270, 10.1007/978-3-030-23201-6_21.

[8] A. W. Chao, K. H. Mess, M. Tigner, and F. Zimmermann, Handbook of Accelerator Physics and Engineering, 2nd ed. (World Scientific, Singapore, 2013).

[9] S. Becker, M. Bussmann, S. Raith, M. Fuchs, R. Weingartner, P. Kunz, W. Lauth, U. Schramm, M. El Ghazaly, F. Grüner, H. Backe, and D. Habs, Characterization and tuning of ultrahigh gradient permanent magnet quadrupoles, Phys. Rev. ST Accel. Beams 12, 102801 (2009).

[10] M. Modena, O. Dunkel, J. G. Perez, C. Petrone, P. A. Thonet, and D. Tommasini, Design, assembly and first measurements of a short model for CLIC final focus hybrid quadrupole QD0, in Proceedings of the 3rd International Particle Accelerator Conference, New Orleans, LA, 2012 (IEEE, Piscataway, NJ, 2012), p. 3.

[11] J. van Tilborg, S. Steinke, C. Geddes, N. Matlis, B. Shaw, A. Gonsalves, J. Huijts, K. Nakamura, J. Daniels, C. Schroeder, C. Benedetti, E. Esarey, S. Bulanov, N. Bobrova, P. Sasorov, and W. Leemans, Active Plasma Lensing for Relativistic Laser-Plasma-Accelerated Electron Beams, Phys. Rev. Lett. 115, 184802 (2015).

[12] W. K. H. Panofsky and W. R. Baker, A focusing device for the external 350Mev proton beam of the 184Inch Cyclotron at Berkeley, Rev. Sci. Instrum. 21, 445 (1950).

[13] C. Lindstrøm, Staging of plasma-wakefield accelerators, Phys. Rev. Accel. Beams 24, 014801 (2021).

[14] M. Migliorati, A. Bacci, C. Benedetti, E. Chiadroni, M. Ferrario, A. Mostacci, L. Palumbo, A. R. Rossi, L. Serafini, and P. Antici, Intrinsic normalized emittance growth in laser-driven electron accelerators, Phys. Rev. ST Accel. Beams 16, 011302 (2013).

[15] D. J. Spence and S. M. Hooker, Investigation of a hydrogen plasma waveguide, Phys. Rev. E 63, 015401(R) (2000).

[16] C. A. Lindstrøm, K. N. Sjobak, E. Adli, J. H. Röckemann, L. Schaper, J. Osterhoff, A. E. Dyson, S. M. Hooker, W. Farabolini, D. Gamba, and R. Corsini, Overview of the CLEAR plasma lens experiment, Nucl. Instrum. Methods Phys. Res., Sect. A 909, 379 (2018).

[17] R. Pompili et al., Focusing of High-Brightness Electron Beams with Active-Plasma Lenses, Phys. Rev. Lett. 121, 174801 (2018).

[18] S. Steinke, J. van Tilborg, C. Benedetti, C. G. R. Geddes, C. B. Schroeder, J. Daniels, K. K. Swanson, A. J. Gonsalves, K. Nakamura, N. H. Matlis, B. H. Shaw, E. Esarey, and W. P. Leemans, Multistage coupling of independent laser-plasma accelerators, Nature (London) 530, 190 (2016).

[19] S. K. Barber, J. H. Bin, A. J. Gonsalves, F. Isono, J. van Tilborg, S. Steinke, K. Nakamura, A. Zingale, N. A. Czapla, D. Schumacher, C. B. Schroeder, C. G. R. Geddes, W. P. Leemans, and E. Esarey, A compact, high resolution energy, and emittance diagnostic for electron beams using active plasma lenses, Appl. Phys. Lett. 116, 234108 (2020).

[20] J. van Tilborg, S. Barber, H.-E. Tsai, K. Swanson, S. Steinke, C. Geddes, A. Gonsalves, C. Schroeder, E. Esarey, S. Bulanov, N. Bobrova, P. Sasorov, and W. Leemans, Nonuniform discharge currents in active plasma lenses, Phys. Rev. Accel. Beams 20, 032803 (2017).
[21] C. A. Lindstrøm and E. Adli, Analytic plasma wakefield limits for active plasma lenses, arXiv:1802.02750.

[22] C. Lindstrøm, E. Adli, G. Boyle, R. Corsini, A. Dyson, W. Farabolini, S. Hooker, M. Meisel, J. Osterhoff, J.-H. Röckemann, L. Schaper, and K. Sjobak, Emittance Preservation in an Aberration-Free Active Plasma Lens, Phys. Rev. Lett. 121, 194801 (2018).

[23] B. Autin, H. Riege, E. Boggasch, K. Frank, L. D. Menna, and G. Miano, A Z-pinch plasma lens for focusing highenergy particles in an accelerator, IEEE Trans. Plasma Sci. 15, 226 (1987).

[24] J. Christiansen, K. Frank, H. Riege, and R. Seeböck, Studies of plasma lens with pseudo-spark geometry for application in high-energy particle accelerators, Technical Report No. 84-10-AA, CERN, Geneva, 1984.

[25] N. A. Bobrova, A. A. Esaulov, J.-I. Sakai, P. V. Sasorov, D. J. Spence, A. Butler, S. M. Hooker, and S. V. Bulanov, Simulations of a hydrogen-filled capillary discharge waveguide, Phys. Rev. E 65, 016407 (2001).

[26] B. H. P. Broks, K. Garloff, and J. J. A. M. van der Mullen, Nonlocal-thermal-equilibrium model of a pulsed capillary discharge waveguide, Phys. Rev. E 71, 016401 (2005).

[27] J.-H. Röckemann, L. Schaper, S. Barber, N. Bobrova, G. Boyle, S. Bulanov, N. Delbos, K. Floettmann, G. Kube, W. Lauth, W. Leemans, V. Libov, A. Maier, M. Meisel, P. Messner, P. Sasorov, C. Schroeder, J. van Tilborg, S. Wesch, and J. Osterhoff, Direct measurement of focusing fields in active plasma lenses, Phys. Rev. Accel. Beams 21, 122801 (2018).

[28] W. H. Bennett, Magnetically self-focussing streams, Phys. Rev. 45, 890 (1934).

[29] E. Adli, Towards awake applications: Electron beam acceleration in a proton driven plasma wake, in Proceedings of IPAC 2016 (JACOW, Geneva, Switzerland, 2016), pp. 2557-2560, 10.18429/JACoW-IPAC2016WEPMY008.

[30] S.-Y. Kim, K. Moon, M. Chung, K. N. Sjobak, E. Adli, S. Doebert, M. Dayyani, E. S. Yoon, I. Nam, and G. Hahn, Witness electron beam injection using an active plasma lens for beam-driven plasma wakefield accelerators, Phys. Rev. Accel. Beams 24, 121304 (2021).

[31] C. Lindstrøm, E. Adli, G. Boyle, R. Corsini, A. Dyson, W. Farabolini, S. Hooker, M. Meisel, J. Osterhoff, J.-H. Röckemann, L. Schaper, and K. Sjobak, Emittance Preservation in an Aberration-Free Active Plasma Lens, Phys. Rev. Lett. 121, 194801 (2018); Phys. Rev. Lett. 122, 129901(E) (2019).

[32] D. Gamba, R. Corsini, S. Curt, S. Doebert, W. Farabolini, G. Mcmonagle, P. Skowronski, F. Tecker, S. Zeeshan, E. Adli, C. Lindstrøm, A. Ross, and L. Wroe, The CLEAR user facility at CERN, Nucl. Instrum. Methods Phys. Res., Sect. A 909, 480 (2018).

[33] R. Corsini, D. Gamba, W. Farabolini, A. Curcio, S. Curt, S. Doebert, R. G. Alia, T. Lefevre, G. McMonagle, P. Skowronski, M. Tali, F. Tecker, E. Adli, C. A. Lindstrøm, K. Sjobaek, A. Lagzda, and R. M. Jones, First experiments at the CLEAR user facility, in Procedings of IPAC2018 (JACOW Publishing, Geneva, Switzerland, Vancouver, BC, 2018) p. 4, 10.18429/JACoW-IPAC2018-THPMF014. 
[34] K. Sjobak et al., Status of the CLEAR electron beam user facility at CERN, in Proceedings of the 10th Int. Particle Accelerator Conf. IPAC2019 (JACoW Publishing, Geneva, Switzerland, 2019), 10.18429/JACoW-IPAC2019MOPTS054.

[35] R. Kieffer, M. Bergamaschi, E. Bravin, W. Farabolini, P. Karataev, T. Lefevre, and S. Mazzoni, Experimental Observation of Shadowing in Optical Transition Radiation, Phys. Rev. Lett. 120, 094802 (2018).

[36] A. E. Dyson, C. Thornton, and S. M. Hooker, A compact, low cost Marx bank for generating capillary discharge plasmas, Rev. Sci. Instrum. 87, 093302 (2016).
[37] C. A. Lindstrøm, Emittance growth and preservation in a plasma-based linear collider, Ph.D. thesis, University of Oslo, 2019.

[38] A. Rosenbluth, R. Garwin, and M. Rosenbluth, Infinite conductivity theory of the pinch, Los Alamos scientific laboratory Technical Report No. 1850, 1955.

[39] J.E. Allen, An Elementary Theory of the Transient Pinched Discharge, Proc. Phys. Soc. London Sect. B 70, 24 (1957).

[40] T. Miyamoto, Analysis of high-density Z-pinches by a snowplow energy equation, Nucl. Fusion 24, 337 (1984). 